\title{
Supporting Creativity Workshops with Interactive Tabletops and Digital Pen and Paper
}

\author{
Florian Geyer, Daniel Klinkhammer, and Harald Reiterer \\ University of Konstanz \\ Human-Computer Interaction Group \\ Box D73, 78457 Konstanz, Germany \\ \{firstname.lastname\}@uni-konstanz.de
}

\begin{abstract}
In this paper we report our findings from an exploratory design study using a combination of an interactive tabletop and digital pen \& paper technology during a full-day creativity workshop with creative professionals. We describe the applied creativity technique, the system design and the employed interaction techniques. The preliminary results of our study show that this combination of interaction modalities introduces a rich design space for creativity support systems and informal design tools.
\end{abstract}

ACM Classification: H5.2 [Information interfaces and presentation]: User Interfaces. - Graphical user interfaces.

General terms: Design, Experimentation

Keywords: Creativity Support, Design Tools, Multi-touch, Interactive Tabletops, Digital Pen \& Paper

\section{INTRODUCTION}

The impact of interactive tabletops on social factors in group work is of emerging interest for creativity support researchers. Tabletops may lower the burden of participation and can stimulate engaging explorations, which are important factors for creativity support tools [1]. In addition to the facilitation of group interaction, the integration of individual content is also an important research issue [2]. For creative problem solving tasks, digital pen \& paper technology provides a natural modality of content creation and integration [3]. We believe that a combination of these technologies can provide an intuitive way of interaction in creative group work. In order to investigate these issues in the field, we conducted an explorative design study during a full-day creativity workshop with creative professionals.

\section{CONTEXT}

The Creativity World Forum is an annual event organized by the Districts of Creativity network that consists of 12 regions from 3 continents [4]. As part of this event, we had the chance to employ a digital tool within a workshop based

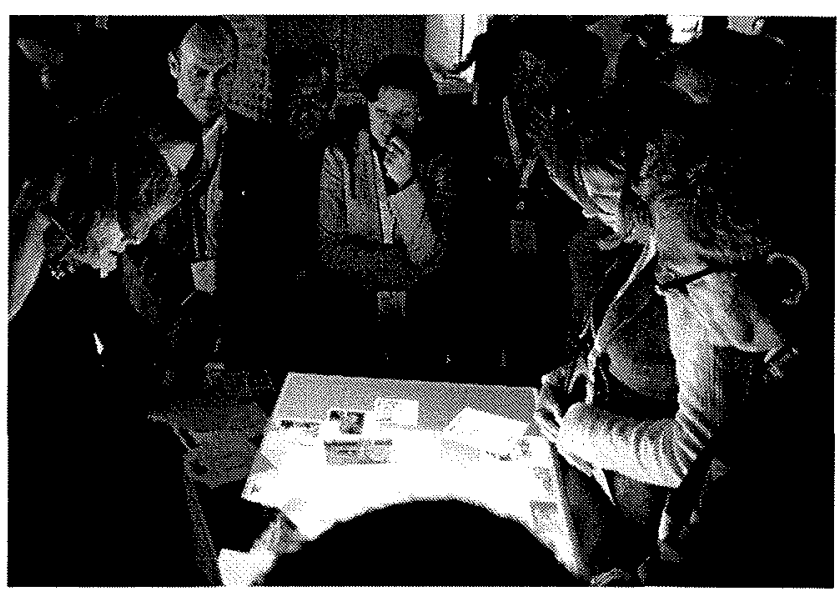

Figure 1: Creative professionals using the system during a breakout session of the creativity workshop

on the creativity technique "The Edison Principle" [5]. Participants of the workshop were professionals from different countries working in creative industries like design, filmmaking, art and music. The creativity method was conducted several times in small groups during break-out sessions. The technique involves specific themes (e.g. tools of productivity), while participants are randomly assigned to different roles (e.g. architect) and tasks (e.g. make something more eco-friendly). The themes are visualized by collections of inspiring images with short captions. Roles and tasks are assigned by picking index cards from a deck. By cooperatively combining the themes with individual roles and tasks, new ideas are created within the group. These ideas are then documented on paper for sharing within the group and across different break-out sessions.

\section{REQUIREMENTS AND SYSTEM DESIGN}

Based on the creativity technique we derived the following requirements for adequate tool support: (a) provide collections of inspirational images from a repository based on specific themes; (b) display images with short captions; (c) support collaborative browsing; (d) support focusing on specific images for group discussion; (e) support creating and adding ideas to the themes by multiple users in parallel (no production blocking); and (f) allow focusing on specific ideas for presentation. 

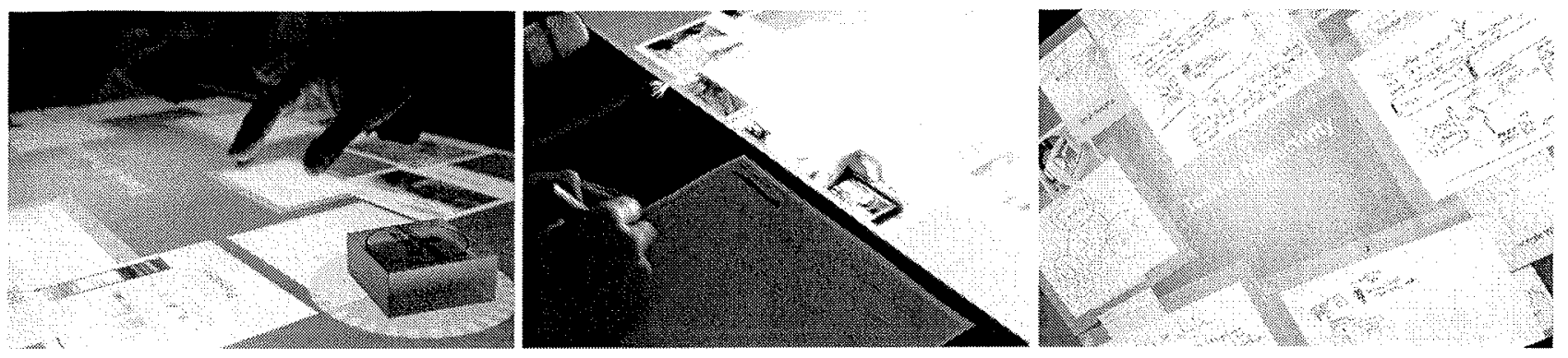

Figure 2: Theme access token (a), digital pen \& paper (b), zoomable pin board with pictures and idea scribbles (c)

Based on these requirements, we designed a system using state-of-the art technology. The technical setup was based on a large, custom-built, 65 inch rear-projection multi-touch table with object recognition and four digital pens from Anoto [6] connected via Bluetooth connections (see Figure 1). We used Microsoft WPF for implementing the interface.

We decided that the basic interface metaphor should be a zoomable pin board where images and idea scribbles were clustered according to the available themes. The view onto the pin board could be navigated by applying zooming and panning gestures, while manipulations (scaling, rotating, flicking) could be used to move and resize objects. We integrated color-coded physical tokens for accessing the different theme clusters and for switching between browsing and presentation mode (see Figure 2, a). By placing a theme token on the surface of the table, the pin board view was zoomed onto the associated theme cluster and manipulation of objects was activated for browsing. When scaling images to larger sizes, a more detailed caption was displayed along with the images (semantic zoom). Idea scribbles could be added to the theme clusters by writing on different pages of paper assigned to each cluster (see Figure 2, b). The pen strokes made on paper were instantly transferred to digital representations within the currently accessed theme cluster (see Figure 2,c). In presentation mode, when no theme token was on the table, the view onto the pin board could also be navigated by tapping gestures that led to an animated zoom onto the selected object.

\section{RESULTS}

During the full-day workshop over 60 creative professionals used our tool. We collected feedback with questionnaires from 16 participants. Additionally, we observed the workshop with 4 researchers and video recordings. From the questionnaires, we found that the participants rated the tool as intuitive and enjoyable and that the overall interaction imposed no burden on the participants. 12 participants stated that they could in general imagine this combination of technology in creative work environments. The best rated aspects (scores based on a 5-point Likert scale) of the system were parallel input (4.5), the zoomable pin board metaphor (4.3) and increased group awareness (4.2). We got mixed answers on the question if the system distracts from the primary task of the group (2.7). However, from our observations we found that the novelty of the applied technologies themselves was distracting for some of the participants, especially in the first sessions. Additionally, we observed several interesting behaviors of the participants such as the following: Participants placed some of our digital pens in their own pockets or tried to write on the paper with regular pens and markers. This indicates that the digital pen was not perceived as technology, but was treated just like a traditional pen. Fortunately, no one tried to write directly on the digital representations of the ideas on the table. In some cases, the theme access token was used for gatekeeping and to negotiate leadership within the groups. Finally, we found that the zooming mechanism is a reasonable tool for focusing attention on specific objects. However, it only works well if one member of the group has the dedicated role of the presenter.

\section{CONCLUSION}

In our design study we explored interaction techniques for supporting creative group activities. We found that the design space for combining digital pen \& paper with interactive tabletops is promising and will therefore continue to investigate these aspects for supporting creativity techniques and design methods.

\section{REFERENCES}

1. Resnick M., Myers B., Nakakoji K., Shneiderman B., Pausch R., Selker T., and Eisenberg M. Design Principles for Tools to Support Creative Thinking. http://www.cs.umd.edu/hcil/CST, 2005.

2. Stewart J., Bederson B. B., Druin A. Single Display Groupware: A Model for Co-present Collaboration. In Proceedings of CHI'99 Human Factors in Computing Systems (Pittsburgh, PA, US), ACM/SIGCHI, NY, 1999, pp. $286-293$

3. Cook D., Bailey B. Designers' Use of Paper and the Implications for Informal Tools. In Proceedings of OZCHI'05 the 17th Australia Conference on ComputerHuman Interaction (Canberra, Australia), CHISIG, AU, 2005. pp. 1-10

4. http://www.districtsofcreativity.org

5. Meyer, J. The Edison Principle: A Brilliantly Simple Path to Successful Ideas. Campus, Frankfurt, DE, 2008.

6. http://www.anoto.com 